FORMATION Formation emploi

Revue française de sciences sociales

113 | janvier-mars 2011

La flexicurité à l'aune de l'approche par les capacités

\title{
Les parcours individuels et la capacité à l'emploi sur les marchés du travail informels : les enseignements de la flexibilité du marché du travail argentin
}

Individuals trajectories and capability-to-work on informal labor markets: lessons from flexibility on the Argentine labor market Individuelle berufliche Laufbahnen und die Fähigkeit, auf informellen Arbeitsmärkten zu arbeiten : Erkenntnisse aus der Flexibilität des argentinischen Arbeitsmarkts

Los itinerarios individuales y la capacidad de trabajar en los mercados de trabajo informales : las enseñanzas de la flexibilidad del mercado de trabajo argentino

\section{Corina Rodríguez Enríquez}

\section{OpenEdition}

Journals

Édition électronique

URL : http://journals.openedition.org/formationemploi/3270

DOI : 10.4000/formationemploi.3270

ISSN : 2107-0946

Éditeur

La Documentation française

Édition imprimée

Date de publication : 1 mars 2011

Pagination : 81-95

ISSN : 0759-6340

Référence électronique

Corina Rodríguez Enríquez, « Les parcours individuels et la capacité à l'emploi sur les marchés du travail informels : les enseignements de la flexibilité du marché du travail argentin », Formation emploi [En ligne], 113 | janvier-mars 2011, mis en ligne le 10 avril 2013, consulté le 30 octobre 2020. URL : http://journals.openedition.org/formationemploi/3270; DOI : https://doi.org/10.4000/

formationemploi.3270 


\title{
DOSSIER
}

\section{Les parcours individuels et la capacité à l'emploi sur les marchés du travail informels: les enseignements de la flexibilité du marché du travail argentin}

Corina Rodríguez Enríquez*

\begin{abstract}
Malgré la différence structurelle entre un marché du travail informel, celui de l'Argentine, et un marché du travail encore très protégé, celui de la France, les pressions vers plus de flexibilité pourraient réduire l'écart entre ces deux pays.
\end{abstract}

Dans les sociétés modernes, le travail rémunéré constitue l'un des éléments structurant de la vie des individus. L'emploi est la voie majeure et la plus valorisée par la société pour obtenir un salaire. L'emploi constitue en outre un moyen de bénéficier de prestations sociales (telles que l'assurance maladie ou la retraite). C'est un espace où le travail humain et les compétences mobilisées pour l'accomplir sont reconnus socialement. C'est aussi un espace où les individus construisent leurs principaux réseaux sociaux.

Ainsi, le rôle économique du travail rémunéré et la reconnaissance sociale de l'activité professionnelle exercée sur le marché du travail (par opposition, par exemple, aux tâches domestiques non rémunérées accomplies au foyer) jouent un rôle majeur. Dès lors, l'emploi est un facteur crucial du développement des capacités individuelles, c'est-à-dire de l'opportunité « d'être » et de « faire $»^{1}$. La situation des individus sur le marché du travail constitue un bon indicateur

1 Pour une étude complète sur la notion de capacité et son évolution, voir Sen (1982, 1985, 1987 et 1992). Pour une explication

* Corina Rodríguez Enríquez est chercheuse au CONICET /Conseil national de la recherche scientifique et technique) et au CIEPP (Centre interdisciplinaire d'étude des politiques publiques) en Argentine. Cet article s'appuie sur les recherches effectuées pour le projet CAPRIGHT (2007-2010). Cynthia Pok et Andrea Lorenzetti faisaient également partie de l'équipe de recherche. 
pour évaluer leur capacité à l'emploi et le développement des compétences.

Le développement de l'État providence nous a conduits à croire que l'emploi permettait d'obtenir un revenu régulier satisfaisant, de bénéficier du filet de protection que représente la sécurité sociale, de faire évoluer sa carrière, de se former et d'acquérir de nouvelles compétences ainsi que d'envisager un présent et un avenir stables et sûrs. Ainsi conçu, l'emploi pouvait (et devait) constituer le meilleur instrument pour améliorer la capacité à l'emploi et les compétences professionnelles. Pour certaines sociétés, ce fut le cas jusqu'à un certain point.

Au sein d'autres sociétés, comme celles d'Amérique latine, une grande partie de la population demeure historiquement exclue de la possibilité d'obtenir un emploi remplissant réellement ces fonctions. Les modèles d'accumulation de ces économies reposent sur la sous-utilisation d'une grande part de la maind'œuvre ${ }^{2}$ qui, face à l'impossibilité d'obtenir un emploi formel, est cantonnée au sous-emploi, à l'emploi informel ou au chômage. Ainsi, l'exercice d'une activité sur le marché du travail a peu de chances de renforcer leurs capacités.

Les réformes structurelles mises en œuvre dans la région, au cours des années 80 , et renforcées dans les années 90, ont modifié les dynamiques du marché $\mathrm{du}$ travail ainsi que les caractéristiques de l'emploi. Cela a engendré un processus de flexibilisation, par l'« informalisation » de ce qui était «formel ». On prétendait alors que ces réformes apporteraient des opportunités d'emploi pour tous.

Cet article se centre sur l'évolution des parcours professionnels, en analysant le cas du marché du travail urbain en Argentine. Il observe plus particulièrement comment la situation macroéconomique influe sur l'activité (et par conséquent sur la capacité à l'emploi), en soulignant les différences entre hommes et femmes.

Il s'agit de savoir comment le rapport au marché du travail façonne les perspectives de vie individuelles

sur la façon dont nous avons intégré ce cadre à notre étude des parcours individuels, voir Rodríguez Enríquez (2008).

${ }^{2}$ C'est l'explication privilégiée par l'école structuraliste latinoaméricaine. Pour une synthèse sur cette question, voir Rodríguez (2006). et quelle est la place de l'emploi parmi les valeurs sociales. Autrement dit, comment concilier les conditions imposées par un marché du travail flexible et informel et les attentes des sociétés vis-à-vis de l'emploi?

La première partie présente brièvement le contexte économique du marché du travail argentin et explique comment la flexibilité du travail s'y caractérise. La seconde partie passe en revue les parcours professionnels individuels des hommes et des femmes, leurs déterminants exogènes (liés à l'environnement macroéconomique) et endogènes (liés aux ressources personnelles). La troisième partie synthétise les principaux résultats et pointe les éléments susceptibles d'éclairer le débat sur la situation européenne.

\section{LE CONTEXTE ARGENTIN ET LE RÔLE DE LA FLEXIBILITÉ ET DE L'INFORMALITÉ SUR LE MARCHÉ DU TRAVAIL}

Historiquement, le marché du travail argentin s'est développé selon les paradigmes d'une croissance sur le long terme, générée par l'industrialisation, avec une tendance au plein emploi, au sein du secteur formel et sur des postes rémunérateurs.

La forte proportion de salariés parmi la population, le faible niveau de sous-emploi de la main-d'œuvre et l'homogénéité relative des revenus que le pays a connus jusqu'aux années 60 en témoignaient. En outre, l'augmentation de l'activité économique des femmes était considérée comme une tendance inévitable accompagnant le processus de modernisation.

Cependant, les limites de cette dynamique sont vite apparues, en particulier avec l'abandon du modèle économique basé sur la substitution à l'importation et avec l'application des mesures de libéralisation économique, à partir des années 70 , intervenue parallèlement à la répression exercée par la dictature militaire sur la population active.

Les événements qui suivirent, engendrés par la concentration des capitaux et la primauté de la valorisation financière et de la désindustrialisation, ont 
compromis la santé et l'homogénéité du marché du travail argentin. Durant cette période : i) le secteur informel urbain se développe; ii) le chômage, souvent sous la forme de l'inactivité, augmente; iii) l'activité dans le secteur formel salarié diminue ; iv) le pouvoir d'achat s'écroule; v) l'activité féminine est cantonnée à certains emplois et ne constitue plus un signe de modernisation, mais une stratégie du ménage pour compléter les revenus de la famille; vi) la couverture sociale est moindre, selon les situations professionnelles.

Dans les années 90, une tentative de consolidation du modèle économique basé sur l'ouverture et la déréglementation des marchés voit le jour. Le cadre institutionnel du marché du travail est totalement transformé, avec de sérieuses répercussions sur la population active. En 1991, après plusieurs années marquées par des périodes d'hyperinflation, une loi est votée afin d'adopter le système de la caisse d'émission monétaire qui lie la monnaie locale au dollar (plan de convertibilité). Grâce au taux de change fixe, l'économie se stabilise et un contexte financier international favorable - conjugué à l'aide des institutions de crédit multilatérales - permet la mise en place d'une série de réformes économiques structurelles.

Suite aux aléas de l'évolution macroéconomique de cette période, le marché du travail est irrégulier, avec une tendance générale à la détérioration ${ }^{3}$. L'une des principales caractéristiques de l'économie, au cours de ces années, est sa faible capacité à générer des emplois, malgré la croissance de la production et de l'investissement.

Le chômage est devenu l'aspect le plus prégnant et le plus inquiétant de la situation économique de la population au cours de ces années. Le phénomène est si répandu qu'il touche toutes les couches de la population, mais ses conséquences ont été plus graves pour les jeunes, les travailleurs peu qualifiés et les chefs de famille.

Une autre caractéristique clé de cette période est la stagnation du secteur informel. Parallèlement, l'informalisation du secteur formel s'accentue. Cela se

\footnotetext{
${ }^{3}$ Beccaria (2005) identifie cinq phases durant cette période : i) une phase d'expansion (1991-1994) ; ii) une phase de récession (19941995); iii) une phase de rétablissement (1996-1998); iv) une nouvelle phase de récession (1998-2001); v) la crise de fin 2001.
}

traduit par une montée de la précarité au sein des entreprises du secteur formel, notamment en raison de la détérioration des conditions de travail et de l'apparition de nouveaux types de contrat, générées par des modifications du droit du travail.

C'est dans ce contexte que l'Argentine adopte la flexibilité ; il est donc essentiel de replacer la réflexion dans ce cadre. Notons d'abord que la flexibilité prend une forme particulière sur les marchés du travail, où l'informalité devient une condition structurellet. Lorsque près de la moitié de la population occupe un emploi non conforme au droit et aux normes du travail et non couvert par la sécurité sociale, la flexibilité devient alors un processus dont les formes légales intègrent de plus en plus les conditions de travail du secteur informel.

Les différentes formes de flexibilité qui se sont diffusées sur le marché du travail sont issues d'une politique visant la réduction des coûts du travail, associée à une diminution des avantages sociaux, et ce afin d'augmenter le taux d'emploi. Le débat amorcé au milieu des années 80 a culminé en 1991, avec la promulgation de la loi sur l'emploi qui a permis un contournement légal des dispositifs de sécurité sociale (Marshall, 1996).

Au-delà de ces moyens légaux, deux types d'actions sont généralement mises en œuvre dans la flexibilisation (Monza, 1996). La première vise à abolir les restrictions à l'entrée et à la sortie du travail (particulièrement à la sortie). Le second type d'action vise à supprimer les restrictions imposées par les accords syndicaux en termes d'obligations, de temps de travail, etc.

On peut discuter de l'efficacité de ces mesures en matière de création d'emplois. Cependant, un consensus existe sur le fait que la flexibilité, particulièrement le premier type, a largement contribué à la rotation et à l'instabilité de la main-d'œuvre, y compris pour les emplois traditionnellement considérés comme stables. La flexibilité apparaît sur ces marchés comme un genre « moderne » d'informalité,

\footnotetext{
${ }^{4}$ Pour une analyse plus détaillée du concept d'informalité sur les marchés du travail d'Amérique latine ainsi que de son évolution historique, voir Giosa Zuazúa (2005). Pour une étude plus spécifique du travail de l'OIT sur l'informalité et les sexes, voir Chant et Pedwell (2008).
} 
créant l'insécurité de l'emploi et la précarisation des conditions de travail. Elle se traduit par une participation discontinue au marché du travail et la dissolution du modèle social du travail (Pok, 1992)

Ainsi, la société a été contaminée par ces «pathologies » du marché du travail. L'aggravation de l'instabilité a gagné les parcours professionnels qui ont oscillé entre inactivité, emploi et chômage.

La récurrence de ce type de parcours et la détérioration de la qualité des emplois due au processus de « flexibilisation/informalisation » ont fragilisé l'aptitude, déjà faible, du marché du travail argentin à constituer un espace d'accomplissement de la capacité à l'emploi et d'acquisition des compétences.

Ces parcours étaient souvent considérés comme des processus individuels, davantage liés aux caractéristiques personnelles de ceux qui les subissaient, qu'à la dynamique d'exclusion et de déstabilisation du marché du travail. Autrement dit, à l'époque, au lieu d'affronter les problèmes, on préférait les imputer aux comportements individuels. En un sens, on estimait que chaque personne était responsable de l'amélioration de sa capacité à l'emploi. Pour résumer, on considérait que le problème résultait d'un manque d'investissement en capital humain ${ }^{5}$.

L'année 2002 est marquée par la fin du plan de convertibilité et la récession économique qui s'ensuit. En 2003, le pays voit le début d'un rétablissement durable de l'activité économique, accompagné d'une croissance de la production, de l'investissement, de la consommation et de l'emploi. Ce contexte macroéconomique a permis d'élaborer un nouveau scénario visant à améliorer le marché du travail. Ce scénario pouvait laisser présager de meilleures opportunités d'emploi pour les hommes et les femmes. Cette promesse s'est-elle traduite concrètement dans les parcours individuels?

\footnotetext{
${ }^{5}$ Ce sont les arguments les plus couramment évoqués pour expliquer la situation des travailleurs peu qualifiés, bien qu'il n'existe aucune preuve absolue d'un quelconque impact positif des programmes de formation sur ce type de population. Nous y reviendrons brièvement dans la partie suivante.
}

\section{LES PARCOURS INDIVIDUELS SUR LES MARCHÉS DU TRAVAIL FLEXIBLES ET INFORMELS}

\section{Analyser les transitions sur le marché de l'emploi}

En s'appuyant sur les données d'une enquête sur les ménages argentins (Encuesta Permanente de Hogares - EPH - INDEC), nous étudions les trajectoires des parcours individuels lors de trois phases macroéconomiques : i) l'expansion économique durant le « plan de convertibilité » (1996-1998), ii) la récession économique durant le «plan de convertibilité » (1999-2001), iii) l'expansion économique après la dévaluation monétaire (2004-2006). Nous identifions, pour chacune de ces périodes, des transitions positives et négatives, selon leur aptitude à favoriser la capacité à l'emploi et le développement des compétences.

La capacité à l'emploi a été évaluée à l'aune de l'activité des personnes (ayant un emploi, au chômage ou inactif). La capacité à développer des compétences a été évaluée à l'aune d'un indicateur de la qualité de l'emploi'.

L'évaluation de la qualité s'avère utile pour apprécier dans quelle mesure l'emploi remplit les fonctions attendues et pour connaître sa propension à favoriser un développement des compétences. Pour cela, nous avons établi deux catégories : i) les emplois de qualité (c'est-à-dire les emplois stables du secteur formel) et ii) les emplois de faible qualité. La dernière catégorie comprend :

- Les employés occupant un emploi temporaire de moins de trois mois ou qui ne bénéficient d'aucun avantage social ou qui, même s'ils en bénéficient, sont sous-employés (travail à temps partiel) quand bien même ils voudraient travailler plus.

- Les travailleurs indépendants qui occupent un emploi temporaire de moins de trois mois ou considéré comme « un petit boulot», soit comme un emploi non qualifié ou ceux qui, bien qu'ils exercent un emploi

${ }^{6}$ Nous supposons qu'un emploi de faible qualité empêche le développement des compétences pour les raisons suivantes : i) l'emploi est de courte durée et n'encourage donc pas la formation; ii) les tâches sont peu qualifiées; iii) le coût relatif de la formation est trop élevé (à cause du faible niveau de productivité). 
qualifié ou professionnel, sont sous-employés (travail à temps partiel) quand bien même ils voudraient travailler plus.

- Les employés de maison.

- Les travailleurs non rémunérés.

Ceux qui ne correspondent à aucune des catégories ci-dessus sont considérés comme occupant un emploi de qualité. La catégorie des emplois de faible qualité permet d'appréhender les diverses formes de travail qui se sont développées sous le paradigme de la flexibilité. Nous partons du principe que dans le contexte d'un marché du travail flexible, la transition vers ce type d'emploi est plus facile et donc plus fréquente.

Nous évaluons ensuite les transitions possibles entre chacun des statuts, comme positives ou négatives. Les transitions positives sont celles qui rapprochent les individus des meilleures formes d'emploi et améliorent leur capacité à l'emploi et leurs compétences. Les transitions négatives éloignent les individus de ce type d'opportunité.

Par conséquent, la transition de l'inactivité au travail est positivement évaluée, sauf si l'individu ne doit pas occuper un emploi salarié, soit parce qu'il est en âge d'être scolarisé (moins de 18 ans) soit parce qu'il a l'âge légal de la retraite. Au contraire, les transitions inverses, comme le passage de l'activité à l'inactivité, pour un individu en âge de travailler, sont évaluées négativement.

Toute transition du chômage vers une forme d'emploi est considérée comme positive, puisqu'elle augmente les chances de développer la capacité à l'emploi. En revanche, toute transition de l'emploi vers le chômage est considérée comme négative.

Toute transition d'un emploi de faible qualité vers un emploi de qualité est considérée comme positive, tandis que la transition inverse est considérée comme négative.

Afin d'analyser la transition entre deux états possibles, une étude fondée sur des comparaisons temporelles a été menée et des matrices de transitions ont été élaborées pour marquer les situations individuelles au début et à la fin de chaque période. Notons qu'EPH est une enquête par panel qui permet de suivre les personnes pendant un an et demi. Dans tous les cas, et afin d'obtenir des échantillons statistiquement significatifs sur la période la plus longue possible, les échantillons ont été élaborés grâce à la mise en commun des différentes vagues pour chaque période ${ }^{7}$. Cette procédure est fréquemment utilisée dans la recherche et permet de présenter simultanément des parcours qui se sont déroulés à des périodes différentes, quoique proches. Au sens strict, nous analysons les transitions entre le début et la fin de chaque période de mise en commun des échantillons plutôt que l'ensemble des événements de cette période ou d'un parcours individuel plus long.

Après avoir déterminé si ces transitions sont positives ou négatives, nous les analysons en fonction de caractéristiques personnelles : âge, niveau d'éducation et responsabilités familiales. Les données sont présentées pour l'ensemble de la population âgée de plus de dix ans, en séparant les hommes et les femmes ${ }^{8}$. Le tableau 1 synthétise les résultats obtenus.

\section{Trajectoires contra cycliques et barrières persistantes}

Si nous considérons qu'une phase d'expansion du cycle économique constitue un scénario propice à la stimulation des capacités liées au marché du travail (capacité à l'emploi et développement des compétences), les transitions suivantes devraient normalement dominer : i) continuité d'un emploi préexistant ; ii) transition d'un emploi de faible qualité vers un emploi de qualité ; iii) transition du chômage ou de l'inactivité vers un emploi, de préférence un emploi qualifié ; iv) peu de transition de l'emploi vers le chômage ou l'inactivité ; v) peu de continuité du chômage.

Pour la première période d'expansion économique, ces anticipations se vérifient dans les parcours individuels ; ces derniers sont en effet nettement marqués par une plus grande continuité de l'emploi pour les emplois de qualité par rapport aux emplois de faible qualité et une transition des emplois de faible qualité vers les emplois de qualité, de même que du chômage vers l'emploi, le plus souvent vers un emploi de faible qualité.

\footnotetext{
${ }^{7}$ Pour les deux premières phases économiques, la plus longue période possible est de quatre ans. Pour la dernière phase, la plus longue période possible est de deux ans et trois mois. Pour plus de détails sur la méthodologie, voir Rodríguez Enríquez (2008).

${ }^{8}$ Pour une analyse plus approfondie des données, voir Rodríguez Enríquez (2009).
} 
En comparant les parcours des hommes et des femmes, on note des similarités et des différences intéressantes.

Premièrement, il y a une bonne continuité de l'emploi durant cette période pour les hommes comme pour les femmes. Bien que les femmes conservent relativement moins les emplois de qualité et plus les emplois de faible qualité que les hommes, la différence reste faible.

Deuxièmement, on constate plus de transitions des emplois de faible qualité vers les emplois de qualité pour les hommes que pour les femmes. La différence est également plus marquée pour les transitions des emplois de faible qualité vers l'inactivité, avec une part plus importante pour les femmes.

Troisièmement, on observe des différences entre les sexes pour les transitions du chômage vers les différents statuts d'emploi. La continuité du chômage est plus marquée pour les hommes $(30 \%)$ que pour les femmes $(23,1 \%)$. Au contraire, la transition du chômage vers l'inactivité est nettement plus importante pour les femmes $(37,8 \%)$ que pour les hommes $(14,9$ \%). Par conséquent, la transition du chômage vers l'emploi durant cette période d'expansion économique est plus souvent le fait des hommes $(55,1 \%)$ que celui des femmes $(39,1 \%)$. De plus, les transitions ne sont pas les mêmes pour les emplois à temps plein. Alors que 40 \% des hommes au chômage retrouvent un emploi à temps plein, c'est le cas pour seulement $27,8 \%$ des femmes.

La période de récession, amorcée en 1998, et qui a perduré après la crise financière qui a caractérisé la fin de l'ère de convertibilité, a eu un impact certain sur le marché du travail et la situation socio-économique de la population. Dans ce contexte, on s'attend à une diminution des parcours individuels positifs, voire même à une inversion vers des parcours négatifs.

Tableau 1

Matrice de transition - Expansion économique - Convertibilité

Octobre 1995 - octobre 1998 - Par sexe. En pourcentage.

\begin{tabular}{|c|c|c|c|c|c|c|}
\hline \multirow{2}{*}{\multicolumn{2}{|c|}{ Total }} & \multicolumn{5}{|c|}{ Statut à la fin de la période } \\
\hline & & \multirow{2}{*}{$\frac{\text { emploi-q }}{76,1}$} & \multirow{2}{*}{$\frac{\text { emploi-f-q }}{14,8}$} & \multirow{2}{*}{$\frac{\text { chômage }}{4,5}$} & \multirow{2}{*}{$\frac{\text { inac }}{4,6}$} & \multirow{2}{*}{$\frac{\text { total }}{100,0}$} \\
\hline \multirow{5}{*}{$\begin{array}{l}\text { Statut au début } \\
\text { de la période }\end{array}$} & emploi-q & & & & & \\
\hline & emploif-q & 23,6 & 51,4 & 9,9 & 15,0 & 100,0 \\
\hline & chômage & 17,1 & 30,9 & 26,9 & 25,1 & 100,0 \\
\hline & inac & 3,0 & 6,8 & 4,9 & 85,4 & 100,0 \\
\hline & total & 26,0 & 17,9 & 7,4 & 48,7 & 100,0 \\
\hline \multirow{2}{*}{\multicolumn{2}{|c|}{ Femmes }} & \multicolumn{5}{|c|}{ Statut à la fin de la période } \\
\hline & & emploi-q & emploi-f-q & chômage & inac & total \\
\hline \multirow{5}{*}{$\begin{array}{l}\text { Statut au début } \\
\text { de la période }\end{array}$} & emploi-q & 74,3 & 14,4 & 2,9 & 8,4 & 100,0 \\
\hline & emploif-q & 16,2 & 53,1 & 8,5 & 22,2 & 100,0 \\
\hline & chômage & 10,9 & 28,2 & 23,1 & 37,8 & 100,0 \\
\hline & inac & 2,4 & 6,4 & 4,5 & 86,7 & 100,0 \\
\hline & total & 15,8 & 15,9 & 6,2 & 62,1 & 100,0 \\
\hline \multirow{2}{*}{\multicolumn{2}{|c|}{ Hommes }} & \multicolumn{5}{|c|}{ Statut à la fin de la période } \\
\hline & & emploi-q & emploi-f-q & chômage & inac & total \\
\hline \multirow{5}{*}{$\begin{array}{l}\text { Statut au début } \\
\text { de la période }\end{array}$} & emploi-q & 76,9 & 14,9 & 5,2 & 2,9 & 100,0 \\
\hline & emploi-f-q & 30,5 & 49,9 & 11,2 & 8,4 & 100,0 \\
\hline & chômage & 22,0 & 33,1 & 30,0 & 14,9 & 100,0 \\
\hline & inac & 4,1 & 7,5 & 5,5 & 82,9 & 100,0 \\
\hline & total & 37,4 & 20,1 & 8,8 & 33,7 & 100,0 \\
\hline
\end{tabular}


Tableau 1 (suite)

Matrice de transition - Récession - Convertibilité

Octobre 1998 - octobre 2001. Par sexe. En pourcentage.

\begin{tabular}{|c|c|c|c|c|c|c|}
\hline \multirow{2}{*}{\multicolumn{2}{|c|}{ Total }} & \multicolumn{5}{|c|}{ Statut à la fin de la période } \\
\hline & & emploi-q & emploi-f-q & chômage & inac & total \\
\hline \multirow{5}{*}{$\begin{array}{l}\text { Statut au début } \\
\text { de la période }\end{array}$} & emploi-q & 74,4 & 15,1 & 5,8 & 4,7 & 100,0 \\
\hline & emploi-f-q & 20,2 & 52,2 & 13,1 & 14,5 & 100,0 \\
\hline & chômage & 13,9 & 27,4 & 32,7 & 26,1 & 100,0 \\
\hline & inac & 2,8 & 7,3 & 5,5 & 84,5 & 100,0 \\
\hline & total & 24,9 & 18,5 & 8,9 & 47,7 & 100,0 \\
\hline \multirow{2}{*}{\multicolumn{2}{|c|}{ Femmes }} & \multicolumn{5}{|c|}{ Statut à la fin de la période } \\
\hline & & emploi-q & emploi-f-q & chômage & inac & total \\
\hline \multirow{5}{*}{$\begin{array}{l}\text { Statut au début } \\
\text { de la période }\end{array}$} & emploi-q & 73,4 & 15,8 & 3,5 & 7,3 & 100,0 \\
\hline & emploi-f-q & 14,0 & 53,9 & 9,9 & 22,3 & 100,0 \\
\hline & chômage & 8,8 & 24,6 & 28,4 & 38,3 & 100,0 \\
\hline & inac & 2,5 & 7,4 & 5,5 & 84,6 & 100,0 \\
\hline & total & 15,9 & 17,1 & 7,4 & 59,6 & 100,0 \\
\hline \multirow{2}{*}{\multicolumn{2}{|c|}{ Hommes }} & \multicolumn{5}{|c|}{ Statut à la fin de la période } \\
\hline & & emploi-q & emploi-f-q & chômage & inac & total \\
\hline \multirow{5}{*}{$\begin{array}{l}\text { Statut au début } \\
\text { de la période }\end{array}$} & emploi-q & 74,9 & 14,7 & 6,9 & 3,5 & 100,0 \\
\hline & emploi-f-q & 25,9 & 50,7 & 16,0 & 7,4 & 100,0 \\
\hline & unemploy & 18,3 & 29,8 & 36,3 & 15,5 & 100,0 \\
\hline & inac & 3,3 & 7,0 & 5,4 & 84,3 & 100,0 \\
\hline & total & 35,1 & 20,2 & 10,6 & 34,1 & 100,0 \\
\hline
\end{tabular}

Matrice de transition - Rétablissement économique - Post-convertibilité 2004 - 2006. Par sexe. En pourcentage.

\begin{tabular}{|c|c|c|c|c|c|c|}
\hline \multirow{2}{*}{\multicolumn{2}{|c|}{ Total }} & \multicolumn{5}{|c|}{ Statut à la fin de la période } \\
\hline & & emploi-q & emploi-f-q & chômage & inac & total \\
\hline \multirow{5}{*}{$\begin{array}{l}\text { Statut au début } \\
\text { de la période }\end{array}$} & emploi-q & 79,3 & 12,7 & 3,0 & 5,0 & 100,0 \\
\hline & emploi-f-g & 24,0 & 52,4 & 8,2 & 15,5 & 100,0 \\
\hline & chômage & 15,2 & 30,1 & 26,9 & 27,7 & 100,0 \\
\hline & inac & 3,7 & 7,4 & 3,9 & 85,1 & 100,0 \\
\hline & total & 29,1 & 19,5 & 6,3 & 45,1 & 100,0 \\
\hline \multirow{2}{*}{\multicolumn{2}{|c|}{ Femmes }} & \multicolumn{5}{|c|}{ Statut à la fin de la période } \\
\hline & & emploi-q & emploi-f-q & chômage & inac & total \\
\hline \multirow{5}{*}{$\begin{array}{l}\text { Statut au début } \\
\text { de la période }\end{array}$} & emploi-q & 76,7 & 13,0 & 2,5 & 7,8 & 100,0 \\
\hline & emploi-f-q & 19,0 & 51,6 & 7,5 & 21,9 & 100,0 \\
\hline & chômage & 10,0 & 24,9 & 25,9 & 39,2 & 100,0 \\
\hline & inac & 3,2 & 7,4 & 3,7 & 85,7 & 100,0 \\
\hline & total & 20,5 & 17,9 & 5,8 & 55,8 & 100,0 \\
\hline \multirow{2}{*}{\multicolumn{2}{|c|}{ Hommes }} & \multicolumn{5}{|c|}{ Statut à la fin de la période } \\
\hline & & emploi-q & emploi-f-q & chômage & inac & total \\
\hline \multirow{5}{*}{$\begin{array}{l}\text { Statut au début } \\
\text { de la période }\end{array}$} & emploi-q & 80,9 & 12,5 & 3,3 & 3,3 & 100,0 \\
\hline & emploi-f-q & 29,0 & 53,2 & 8,8 & 8,9 & 100,0 \\
\hline & chômage & 20,6 & 35,5 & 27,9 & 15,9 & 100,0 \\
\hline & inac & 4,6 & 7,3 & 4,3 & 83,8 & 100,0 \\
\hline & total & 39,0 & 21,3 & 6,8 & 32,8 & 100,0 \\
\hline
\end{tabular}

Source : basée sur l'enquête EPH.

emploi-q = emploi de qualité ; emploi-f-q = emploi de faible qualité. 
En effet, le contexte économique défavorable doit en théorie influer sur les parcours individuels de la manière suivante : i) une faible continuité de l'emploi ; ii) plus de transitions des emplois qualifiés vers les emplois de faible qualité ; iii) plus de transitions de l'emploi vers le chômage ou l'inactivité ; iv) moins de transitions du chômage et de l'inactivité vers l'emploi ; v) une plus forte continuité du chômage.

Ces tendances sont confirmées par les données. Durant la période de récession, la continuité de l'emploi recule : 74,4\% des individus conservent des emplois de qualité et $52,2 \%$ des emplois de faible qualité. Simultanément, la transition d'un emploi de faible qualité vers un emploi de qualité chute à 20,2\%.

Cette période est marquée par la hausse des situations de continuité du chômage et la baisse des transitions du chômage vers l'emploi. La transition du chômage vers l'inactivité stagne pour l'ensemble de la population.

Ces tendances des parcours professionnels observées durant la période de récession touchent aussi bien les hommes que les femmes. Dans les deux cas, l'augmentation de la continuité du chômage et la diminution de la continuité de l'emploi (principalement l'emploi de qualité) sont proportionnelles. De plus, il n'y a pas de modification du volume de transitions d'un emploi de qualité vers un emploi de faible qualité entre les périodes d'expansion et de récession pour les hommes comme pour les femmes. Cependant, la transition directe de l'emploi vers le chômage gagne du terrain. Par conséquent, la hausse relative du chômage indique avec certitude la présence d'un mécanisme de réajustement, tandis que le recours à l'emploi de faible qualité en tant qu'alternative de secours régresse.

Soulignons deux différences entre les parcours masculins et féminins. Premièrement, la diminution des passages de la main-d'œuvre d'emplois de faible qualité vers des emplois de qualité affecte davantage les hommes que les femmes. Deuxièmement, la hausse des transitions de l'emploi de faible qualité vers le chômage est également relativement plus marquée pour les hommes que pour les femmes durant cette période, comparée à la précédente.

Enfin, la période de récession ne comporte pas plus de transitions de l'activité vers l'inactivité et ce aussi bien pour les hommes que pour les femmes. Sans doute qu'un contexte économique peu favorable n'incite pas les individus à sortir du marché du travail. En effet, il est probable que les stratégies de survie des ménages visent à maintenir en leur sein le même nombre d'individus actifs et à compenser la dégradation du statut et du revenu du travail.

Le rétablissement économique qui a suivi la période de convertibilité a permis une amélioration du marché du travail, notamment un regain de l'emploi et un recul significatif de l'emploi de faible qualité, même si la qualité des emplois créés était discutable et leur accès quelque peu inégal.

Dans ce contexte, on peut s'attendre à des répercussions positives sur les parcours individuels, dont l'analyse doit révéler quels groupes de population ont été favorisés par cette nouvelle situation.

Les tendances durant cette période sont similaires à celles constatées pendant la période d'expansion économique de la phase de convertibilité. L'observation des parcours professionnels confirme qu'un contexte d'expansion économique favorise les transitions vers l'emploi ou sa continuité et fait reculer les transitions vers le chômage ou sa continuité.

Les données montrent que la continuité de l'emploi est élevée et que plus l'emploi est de bonne qualité au début de la période, plus il est susceptible de perdurer. Le faible taux de continuité des emplois de faible qualité s'explique par le poids des transitions vers des emplois de qualité. Grâce à l'essor des opportunités d'emploi, presque la moitié de la population (45,3\%) au chômage au début de la période de rétablissement économique a trouvé un emploi. Au cours au moins des premières années de cette période, la plus forte augmentation de création d'emplois a concerné les emplois de faible qualité. En effet, tandis que $30 \%$ des individus au chômage ont trouvé un emploi de faible qualité, ce chiffre chute à $15,2 \%$ pour les emplois de qualité.

L'analyse distincte des parcours masculins et féminins révèle des différences significatives. Premièrement, la continuité de l'emploi est relativement plus prononcée pour les hommes, et cette différence s'accentue encore pour les emplois de qualité. Deuxièmement, le passage à un meilleur emploi concerne plus souvent les hommes que les femmes. Troisièmement, la 
continuité du chômage est plus importante pour les hommes, bien que cette différence soit considérablement minorée par rapport à la période de récession des dernières années du régime de convertibilité.

Il est intéressant d'observer que les femmes ont connu plus de transitions vers l'inactivité durant la période de rétablissement économique qui a suivi la phase de convertibilité, non seulement par rapport à la période de récession précédente, mais aussi par rapport à la période d'expansion économique de la phase de convertibilité. Cela témoigne de l'incapacité d'un contexte macro-économique favorable et d'une meilleure situation générale du marché du travail à influer sur cette tendance. En outre, une telle continuité montre que la main-d'œuvre féminine constitue une « réserve » qui entre sur le marché du travail durant les périodes de récession afin de compenser la détérioration de statut et de revenu du travail. Une fois que les conditions s'améliorent, les travailleuses de réserve retournent vers l'inactivité.

Cela a un fort impact du point de vue des capacités individuelles. En effet, on est en droit de se demander s'il faut considérer comme négatif le fait qu'une femme avec un emploi précaire, qu'elle avait pris pour compenser la réduction des revenus du ménage, quitte son emploi et retourne à l'inactivité lorsque les revenus du ménage retrouvent leur niveau antérieur. On peut aussi considérer que cette décision est tout à fait logique. Étant donné la faiblesse de sa rémunération et le rétablissement des revenus du ménage, il est plus pratique pour la femme de se consacrer à nouveau exclusivement au soin des enfants. Cette transition n'est donc pas en soi négative; elle peut même être considérée comme la décision la plus raisonnable étant donné les circonstances.

Cependant, concernant l'enjeu qui nous intéresse ici, le retour à l'inactivité ou l'abandon du marché du travail constitue une transition négative dans la mesure où le marché du travail est un mécanisme socialement valorisé de développement de la capacité à l'emploi et des compétences. Ces transitions ne sont pas négatives en soi, mais elles reflètent le manque d'options qui s'offrent à ces femmes : emploi précaire ou inactivité.

Ces transitions sont aussi négatives car elles mettent au jour les facteurs restrictifs du choix des femmes : la discrimination sur le marché du travail, le manque de services de garde et une répartition inégale des tâches domestiques au sein du ménage. C'est une transition négative dans la mesure où elle fait de l'entrée de la femme sur le marché du travail un sous-produit de l'emploi de son conjoint, ce qui porte atteinte à son autonomie.

C'est aussi une transition négative car les ménages envisagent rarement la possibilité que l'homme, plutôt que la femme, retourne à l'inactivité, dans la mesure où les hommes sont susceptibles de trouver de meilleures opportunités sur le marché du travail, et aussi parce que l'idée que la femme est naturellement disposée à prendre soin des enfants est profondément ancrée dans les esprits. Le caractère injuste de ces préjugés nous incite à considérer ce type de transition comme négative.

Est-ce que les caractéristiques personnelles sont pertinentes pour éclairer les transitions positives ou négatives dans les différentes phases économiques? Le tableau 2 synthétise les résultats relatifs à cette interrogation.

Pour chacune des trois périodes, un nombre plus important de femmes connaît un parcours professionnel négatif. Les jeunes subissent le plus de transitions négatives sur le marché du travail. Il existe une différence notable en termes d'accès au marché du travail entre les hommes et les femmes d'âge moyen. Cette différence s'explique par le fait qu'un plus grand nombre de femmes appartenant à ce groupe d'âge restent inactives (ce qui est considéré dans ce contexte comme un parcours négatif) et qu'un nombre élevé d'hommes passent du chômage ou d'un emploi de faible qualité à un emploi de qualité (ce qui est considéré comme un parcours positif).

L'existence d'une relation positive entre le niveau d'éducation et la qualité de l'emploi est, comme l'on pouvait s'y attendre, vérifiée. On peut noter cependant qu'elle est plus marquée pour les hommes que pour les femmes. Dans le cas des femmes, l'effet positif de l'éducation est plus évident à un niveau d'éducation supérieur. Plus le niveau d'éducation est bas, plus les femmes sont inactives. Quant à la relation entre le type de parcours professionnels et les responsabilités familiales, on observe que les femmes ayant des enfants de moins de six ans connaissent proportionnellement 
plus de parcours négatifs, tandis que la proportion de parcours positifs et négatifs est tout à fait semblable entre les hommes n'ayant pas d'enfant de moins de six ans et ceux qui en ont plus d'un.
Durant les périodes de ralentissement économique, les parcours professionnels des hommes se dégradent plus que ceux des femmes ; cependant, c'est parmi les femmes que l'on enregistre la plus forte valeur

Tableau 2

Parcours professionnels par sexe et type de transition, groupe d'âge, niveau d'éducation et enfant à charge.

\begin{tabular}{|c|c|c|c|c|c|c|}
\hline & \multirow{2}{*}{\multicolumn{2}{|c|}{ Oct 1995 - oct 1998}} & \multirow{2}{*}{\multicolumn{2}{|c|}{ Oct 1998 - oct 2001}} & \multirow{2}{*}{\multicolumn{2}{|c|}{ Début 2004 à début 2006}} \\
\hline & & & & & & \\
\hline & \multicolumn{2}{|c|}{ Total } & \multicolumn{2}{|c|}{ Total } & \multicolumn{2}{|c|}{ Total } \\
\hline & $\begin{array}{c}\text { Transitions } \\
\text { positives }\end{array}$ & $\begin{array}{l}\text { Transitions } \\
\text { négatives }\end{array}$ & $\begin{array}{c}\text { Transitions } \\
\text { positives }\end{array}$ & $\begin{array}{l}\text { Transitions } \\
\text { négatives }\end{array}$ & $\begin{array}{c}\text { Transitions } \\
\text { positives }\end{array}$ & $\begin{array}{l}\text { Transitions } \\
\text { négatives }\end{array}$ \\
\hline Total & 45,0 & 55,0 & 43,6 & 56,4 & 48,4 & 51,6 \\
\hline \multicolumn{7}{|l|}{$\hat{A} g e$} \\
\hline Moins de 25 & 38,7 & 61,3 & 36,2 & 63,8 & 39,5 & 60,5 \\
\hline Entre 25 et 45 & 51,7 & 48,3 & 49,3 & 50,7 & 54,5 & 45,5 \\
\hline Plus de 45 & 41,4 & 58,6 & 42,1 & 57,9 & 47,4 & 52,6 \\
\hline \multicolumn{7}{|l|}{ Niveau d'éducation } \\
\hline Primaire & 35,5 & 64,5 & 33,2 & 66,8 & 39,4 & 60,6 \\
\hline Secondaire & 46,8 & 53,2 & 44,0 & 56,0 & 46,4 & 53,6 \\
\hline Supérieur & 59,0 & 41,0 & 57,1 & 42,9 & 60,5 & 39,5 \\
\hline \multicolumn{7}{|c|}{ Enfant de moins de six ans } \\
\hline Aucun & 45,5 & 54,5 & 44,5 & 55,5 & 48,8 & 51,2 \\
\hline Un & 45,6 & 54,4 & 43,3 & 56,7 & 49,7 & 50,3 \\
\hline \multirow[t]{3}{*}{ Plus d'un } & 40,8 & 59,2 & 37,6 & 62,4 & 42,4 & 57,6 \\
\hline & \multicolumn{2}{|c|}{ Femmes } & \multicolumn{2}{|c|}{ Femmes } & \multicolumn{2}{|c|}{ Femmes } \\
\hline & $\begin{array}{c}\text { Transitions } \\
\text { positives }\end{array}$ & $\begin{array}{l}\text { Transitions } \\
\text { négatives }\end{array}$ & $\begin{array}{c}\text { Transitions } \\
\text { positives }\end{array}$ & $\begin{array}{l}\text { Transitions } \\
\text { négatives }\end{array}$ & $\begin{array}{c}\text { Transitions } \\
\text { positives }\end{array}$ & $\begin{array}{l}\text { Transitions } \\
\text { négatives } \\
\end{array}$ \\
\hline Total & 34,1 & 65,9 & 34,9 & 65,1 & 39,8 & 60,2 \\
\hline \multicolumn{7}{|l|}{ Âge } \\
\hline Moins de 25 & 34,9 & 65,1 & 33,8 & 66,2 & 34,9 & 65,1 \\
\hline Entre 25 et 45 & 37,6 & 62,4 & 37,5 & 62,5 & 44,1 & 55,9 \\
\hline Plus de 45 & 28,3 & 71,7 & 32,2 & 67,8 & 37,7 & 62,3 \\
\hline \multicolumn{7}{|l|}{ Niveau d'éducation } \\
\hline Primaire & 21,6 & 78,4 & 22,3 & 77,7 & 27,1 & 72,9 \\
\hline Secondaire & 33,3 & 66,7 & 34,3 & 65,7 & 35,2 & 64,8 \\
\hline Supérieur & 54,9 & 45,1 & 50,3 & 49,7 & 56,6 & 43,4 \\
\hline \multicolumn{7}{|c|}{ Enfant de moins de six ans } \\
\hline Aucun & 36,3 & 63,7 & 37,2 & 62,8 & 41,3 & 58,7 \\
\hline Un & 30,4 & 69,6 & 31,7 & 68,3 & 38,0 & 62,0 \\
\hline Plus d'un & 27,0 & 73,0 & 25,4 & 74,6 & 31,6 & 68,4 \\
\hline
\end{tabular}


Tableau 2 (suite)

\begin{tabular}{|c|c|c|c|c|c|c|}
\hline & \multicolumn{2}{|c|}{ Hommes } & \multicolumn{2}{|c|}{ Hommes } & \multicolumn{2}{|c|}{ Hommes } \\
\hline & $\begin{array}{l}\text { Transitions } \\
\text { positives }\end{array}$ & $\begin{array}{c}\text { Transitions } \\
\text { négatives }\end{array}$ & $\begin{array}{c}\text { Transitions } \\
\text { positives }\end{array}$ & $\begin{array}{l}\text { Transitions } \\
\text { négatives }\end{array}$ & $\begin{array}{c}\text { Transitions } \\
\text { positives }\end{array}$ & $\begin{array}{c}\text { Transitions } \\
\text { négatives }\end{array}$ \\
\hline Total & 56,0 & 44,0 & 52,7 & 47,3 & 57,4 & 42,6 \\
\hline \multicolumn{7}{|l|}{$\hat{\text { Âge }}$} \\
\hline Moins de 25 & 42,1 & 57,9 & 38,6 & 61,4 & 43,8 & 56,2 \\
\hline Entre 25 et 45 & 67,5 & 32,5 & 63,5 & 36,5 & 66,7 & 33,3 \\
\hline Plus de 45 & 53,5 & 46,5 & 51,5 & 48,5 & 57,1 & 42,9 \\
\hline \multicolumn{7}{|l|}{ Niveau d'éducation } \\
\hline Primaire & 48,5 & 51,5 & 43,7 & 56,3 & 51,0 & 49,0 \\
\hline Secondaire & 59,5 & 40,5 & 53,3 & 46,7 & 57,3 & 42,7 \\
\hline Supérieur & 64,2 & 35,8 & 66,3 & 33,7 & 65,5 & 34,5 \\
\hline \multicolumn{7}{|c|}{ Enfant de moins de six ans } \\
\hline Aucun & 54,3 & 45,7 & 51,9 & 48,1 & 56,3 & 43,7 \\
\hline Un & 62,6 & 37,4 & 56,3 & 43,7 & 63,1 & 36,9 \\
\hline Plus d'un & 55,8 & 44,2 & 51,5 & 48,5 & 54,8 & 45,2 \\
\hline
\end{tabular}

Source : basée sur l'enquête EPH.

Exemple de lecture : parmi les femmes qui occupaient un emploi de qualité en 1995, 76,1\% occupent, en 1998, un emploi de qualité, tandis que $14,8 \%$ occupent un emploi de faible qualité, que $4,5 \%$ sont au chômage et que 4,6 \% sont inactives. Sont aussi présentés les résultats pour les hommes et pour le total, hommes et femmes, et ce pour les trois périodes considérées.

relative de parcours négatifs. Le nombre relatif de parcours positifs et négatifs reste similaire pour les femmes durant les périodes de récession et d'expansion économique. Cela s'explique, comme nous l'avons vu, par le rôle de réserve ou de " tampon » joué par la main-d'œuvre féminine pendant les périodes de récession; elles passent de l'inactivité à l'activité (la transition est considérée comme positive dans notre analyse) afin de compenser la baisse du revenu familial due à une détérioration du marché du travail.

Les parcours positifs restent majoritaires pour les femmes ayant un niveau d'éducation supérieur, mais ils accusent une baisse plus importante que pour les hommes. Les parcours négatifs de femme ayant plus de responsabilités familiales dominent, sans que l'on observe de variation significative entre les périodes de récession et d'expansion économique. Cela reflète combien les parcours professionnels des femmes sont indépendants de la situation économique. Parmi les hommes, l'augmentation des parcours négatifs affecte particulièrement les jeunes et les individus ayant un faible niveau d'éducation ; les parcours professionnels d'hommes ayant des enfants à charge et de ceux qui n'en ont pas sont assez homogènes. Ces différences sont moins marquées que celles constatées pour les femmes.

La relation entre les caractéristiques personnelles des individus et leur parcours professionnel durant la seconde période de rétablissement économique suit les mêmes tendances que celles analysées pour la période précédente. Durant cette période, on observe un lien encore plus étroit entre niveau d'éducation et parcours professionnel. Les parcours professionnels des femmes restent davantage conditionnés par leurs responsabilités familiales que ceux des hommes, bien que cette tendance semble légèrement moins marquée par rapport aux périodes précédentes.

Pour résumer, les données montrent qu'un contexte macro-économique favorable ne suffit pas à bousculer les stratifications des parcours professionnels et à modifier les possibilités offertes aux individus en fonction de leurs caractéristiques personnelles (âge, sexe, niveau d'éducation, responsabilités familiales). Durant la période de rétablissement économique qui suit la phase de convertibilité, les jeunes (et plus 
Tableau 3

Parcours professionnels des bénéficiaires du programme PJJHD Taux de transition de début 2004 à début 2006 - Par sexe en \%

\begin{tabular}{|l|c|c|c|c|c|c|}
\cline { 2 - 7 } \multicolumn{1}{c|}{} & \multicolumn{5}{c|}{ Situation des bénéficiaires du PJJHD à la fin de la période } \\
\cline { 2 - 7 } \multicolumn{1}{c|}{} & Emploi-q & Emploi-f-q & PJHD & Chômage & Inactivité & Total \\
\hline Total & 6,7 & 17,0 & 49,0 & 7,0 & 20,3 & 100,0 \\
\hline Femmes & 4,7 & 12,8 & 50,1 & 7,1 & 25,3 & 100,0 \\
\hline Hommes & 13,4 & 30,8 & 45,6 & 6,6 & 3,6 & 100,0 \\
\hline
\end{tabular}

Source : basée sur les données EPH.

PJJHD : Plan pour les chefs de famille au chômage.

Emploi-q : emploi de qualité. Emploi-f-q : emploi de faible qualité.

particulièrement les jeunes femmes) et les femmes ayant plus de responsabilités familiales connaissent proportionnellement plus de parcours professionnels négatifs.

\section{Les programmes de formation améliorent-ils les parcours professionnels et la capacité à l'emploi ?}

Les politiques publiques argentines se sont emparées de la question des parcours professionnels dès le milieu des années 90 . Les programmes sociaux mis en œuvre se sont concentrés sur deux objectifs : i) soutenir les revenus individuels et ii) améliorer les opportunités sur le marché du travail. La formation était considérée comme une question majeure pour le deuxième objectif. Les femmes faisaient partie des publics cibles de ce programme.

Le plan pour les chefs de famille au chômage (Plan Jefes y Jefas de Hogar Desocupados ou PJJHD) a constitué le programme phare de cette période et combinait les deux objectifs. Il a été initié en 2002, au cours d'une crise économique profonde (durant laquelle le taux de pauvreté ${ }^{9}$ a atteint $54 \%$ de la population) pour les chefs de ménage au chômage ayant des enfants de moins de 18 ans. Les bénéficiaires devaient exercer une activité pour percevoir l'allocation, ce qui était censé favoriser le renouvellement des compétences et améliorer la capacité à l'emploi.

9 Le taux de pauvreté est le pourcentage de ménages dont le revenu total se situe en dessous du seuil de pauvreté, défini comme le niveau de ressources financières mensuelles permettant d'acheter le panier minimum (de base) alimentaire et non alimentaire.
Le programme a bénéficié à prés de deux millions de personnes, dont $70 \%$ de femmes.

Afin de déterminer l'efficacité du programme dans l'amélioration de la capacité à l'emploi et le développement des compétences, les parcours professionnels des bénéficiaires sont analysés dans le tableau $3^{10}$.

Les données montrent que $50 \%$ des bénéficiaires du PJJHD sont sortis du programme à la fin de la période. Près de la moitié de ceux qui l'ont quitté sont entrés sur le marché du travail ; cependant, la plupart ont obtenu un emploi de faible qualité. $7 \%$ des personnes bénéficiaires du programme sont encore au chômage.

Les différences entre les sexes sont criantes, en particulier pour la transition entre le plan PJJHD et l'inactivité. Alors que cette transition intervient très rarement pour les hommes $(3,6 \%)$, un quart des femmes (25,3\%) bénéficiaires du plan au début de la période sont inactives à la fin de la période.

Les transitions du programme PJJHD vers l'inactivité correspondent aux transitions observées dans l'ensemble de la population active pour les femmes sous statut précaire (au chômage ou ayant un emploi de faible qualité), à faible niveau d'éducation et avec plus de responsabilités familiales.

Par conséquent, dans ces cas, l'inactivité des femmes s'explique par une combinaison d'éléments : i) les meilleures opportunités d'emplois sont le plus souvent saisies par les hommes ; ii) le manque d'opportunités

${ }^{10}$ Nos sources ne nous permettent pas de distinguer entre le plan PJJHD et d'autres plans d'aide à l'emploi. Étant donné l'ampleur de ce plan, il est très probable que la plupart des individus visés soient effectivement bénéficiaires du programme. 
pour ce groupe de population (les femmes ayant le profil sociodémographique des bénéficiaires du PJJHD) ; iii) l'absence d'infrastructure ou de système d'accueil pour la petite enfance permettant de faire face aux tâches domestiques et au travail.

Pour résumer, l'analyse du PJJHD révèle que ce type de programme de formation n'améliore pas significativement les opportunités de travail pour les femmes pauvres. L'activité des bénéficiaires ne s'est guère améliorée. Le volet formation du programme a peiné à offrir des opportunités de développement de la capacité à l'emploi (en particulier pour les femmes pauvres). Les difficultés à acquérir de nouvelles et de meilleures compétences, ainsi qu'à les utiliser dans un travail, freinent la capacité des femmes pauvres à développer leurs compétences.

Ces données corroborent les recherches sur les difficultés rencontrées par les travailleurs peu qualifiés, en particulier les jeunes et les femmes, pour entrer sur le marché du travail ${ }^{11}$. Ainsi, les obstacles liés au manque de ressources personnelles (manque d'éducation, de formation, de compétences, charges familiales conséquentes ou assumées à un jeune âge) associés à la dynamique du marché du travail expliquent les problèmes que rencontre ce public pour améliorer sa capacité à l'emploi.

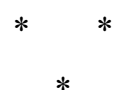

En termes de parcours professionnels et de capacités, le cas argentin est-il si éloigné de la situation européenne?

Afin de proposer quelques réponses à cette interrogation, revenons d'abord sur les principales conclusions que nous pouvons tirer du cas argentin :

- Le contexte macro-économique permet d'expliquer la dynamique du marché du travail.

- Les caractéristiques structurelles du marché du travail influencent sa propension à développer la capacité à l'emploi et les compétences.

- Le fort taux d'emplois de faible qualité (dans le secteur informel, avec des salaires faibles, des tâches

${ }^{11}$ Pour un ouvrage en français sur la situation des jeunes femmes en Argentine, voir Millenaar (2009). peu qualifiées et une faible couverture sociale) creuse le fossé entre la capacité à l'emploi et la capacité à développer des compétences (ou les autres capacités liées au travail). Bien que présents sur le marché $\mathrm{du}$ travail, les individus peinent à améliorer leurs compétences.

- L'informalisation, comme processus de réajustement du marché du travail, augmente la part de parcours professionnels négatifs (de l'emploi vers l'emploi de faible qualité ou vers l'inactivité). Plus le parcours est négatif, moins il est susceptible d'améliorer la capacité à l'emploi et de développer les compétences.

- Le processus de flexibilisation du marché du travail implique l'adoption, par certains types d'emploi du secteur formel, des caractéristiques du secteur informel, ce qui limite leur aptitude à améliorer les capacités.

- Les travailleurs peu qualifiés, les jeunes et les femmes se heurtent à des obstacles majeurs pour améliorer leur capacité à l'emploi et leurs compétences. Interviennent ici deux facteurs : le manque de ressources personnelles et la dynamique du marché du travail, discriminatoire à l'égard de ces publics (comme en témoigne leur surreprésentation dans les emplois de faible qualité, le chômage et l'inactivité).

- Bien que la formation proposée par le PJJHD (plan de formation des chefs de foyer au chômage) puisse être utile, elle est insuffisante pour surmonter les difficultés évoquées plus haut.

- Les responsabilités familiales (en l'absence de services de garde et du partage des tâches entre hommes et femmes) semblent être l'un des principaux obstacles au développement de la capacité à l'emploi et des compétences, pour les femmes, et en particulier les femmes pauvres ou peu qualifiées.

Certes, Buenos Aires est loin de Paris et des autres capitales européennes et la plupart des marchés du travail européens ne ressemblent pas à celui de l'Argentine ou ceux d'autres pays d'Amérique latine, caractérisés par le poids du secteur informel ou l'absence de protection sociale. Cependant, certains phénomènes et certaines questions qui sont soulevées en France et en Europe ne sont sans doute pas si éloignés du cas argentin. En voici quelques exemples : 
- La crise mondiale actuelle permet aux chantres de plus de flexibilité de mieux se faire entendre. Simultanément, les travailleurs estiment que leurs conditions de travail se dégradent (Méhaut, 2008). Cela révèle une baisse de la qualité de l'emploi sur les marchés du travail français et européen.

- Les jeunes européens et les jeunes français rencontrent de plus en plus d'obstacles à l'entrée sur le marché du travail et la probabilité qu'ils trouvent un emploi peu protégé, instable et précaire est forte. De plus, les contrats non standards semblent être devenus quasiment la norme lors de l'entrée sur le marché du travail des jeunes européens (Moncel et Sultzer, 2006).

- En Europe, les parcours professionnels des travailleurs peu qualifiés (principalement les femmes) sont instables, fragiles, peu sécurisés, avec de faibles perspectives d'évolution (Méda et Vennat, 2004). Ils sont par conséquent peu aptes à favoriser la capacité à l'emploi et le développement des compétences.

- Les femmes ayant plus de responsabilités familiales doivent se contenter des opportunités de travail les moins intéressantes (principalement les emplois à temps partiel, les moins qualifiés, les moins bien payés et les moins protégés), ce qui restreint leurs capacités en matière de travail. La capacité à l'emploi est encore plus faible pour les femmes ayant plus d'un enfant de moins de trois ans. (Mehaut, 2008).
- Les travailleurs migrants, en France et en Europe, subissent des discriminations et se heurtent à de nombreux obstacles dans l'amélioration de leur capacité à l'emploi et le développement de leurs compétences.

En résumé, l'expérience du travail, pour certains groupes de population en France et en Europe, ne semble pas si éloignée du portrait que nous avons brossé pour l'Argentine. Malgré la différence structurelle entre un marché du travail informel (celui de l'Argentine) et un marché du travail encore très protégé (celui de la France), les pressions vers plus de flexibilité pourraient réduire l'écart entre les deux pays.

En un sens, l'expérience vécue par l'Argentine peut éclairer certaines questions du débat européen, notamment l'attention particulière qu'il convient d'accorder à certains groupes de population, dans la mesure où ils ne sont pas responsables des difficultés qu'ils rencontrent dans le développement de leur capacité à l'emploi. Cependant, il s'agit de la conséquence d'une dynamique d'ensemble qui ne peut être modifiée uniquement par des politiques du marché du travail ciblées.

Traduction de l'anglais au français : Camille Richou, relue par M. Zangani

\section{Bibliographie}

Chant S. et Pedwell C. (2008), Women, gender and the informal economy: An assessment of ILO research and suggested ways forward. Genève : ILO.

Giosa Zuazúa N. (2005), « De la marginalidad y la informalidad, como excedente de fuerza de trabajo, al empleo precario y al desempleo como norma de crecimiento », Buenos Aires : Ciepp. DT 47.

Marshall A. (1996), «Protección del empleo en América Latina: las reformas de los 90 y sus efectos en el Mercado de trabajo ", Estudios del Trabajo 11.
Méda D. et Vennat F. (2004), Le travail non qualifié : permanences et paradoxes, Éditions La Découverte.

Mehaut Ph. (2008), Decent Work Contry Report, Genève : ILO.

Millenaar V. (2009), « L'insertion professionnelle des jeunes femmes. Un défi pour les politiques de formation professionnelle », Chronique Internationale de L'Ires, 119, pp. 32-39.

Moncel N. et Sulzer E. (eds) (2006), «L'intégration des jeunes opérateurs chez PSA. Contextes sociétaux, 
effets de site et identitiés au travail », Marseille, Cereq, Notes Emploi Formation 22.

Monza A. (1996), «Flexibilidad y empleo », Coyuntura y Desarrollo, 217.

Pok C. (1992), « Precariedad laboral: personificaciones sociales en la frontera de la estructura del empleo », Paper prepared for the Inter-American Seminar on Measurement of Informa Employment, OAS/INEI.

Rodríguez O. (2006), El estructuralismo latinoamericano, Santiago, Cepal.

Rodríguez Enríquez C. (2008), Causas y Azares. Trayectorias ocupacionales, asistencialismo y oportunidades de vida de mujeres y varones en Argentina, Buenos Aires, FLACSO.
Rodríguez Enríquez C. (2009), "Labour trajectories and social assistance policies in flexible labour makerts. Insights from Argentina", In Vladimirova, K. (ed.) Transformations in Labour: Social Security and Social Dialogue, Sofia : Ciela.

Sen A. (1982) Choice, Welfare and Measurement. Oxford : Blackwell.

Sen A. (1985) Commodities and Capabilities. Amsterdam : North-Holland.

Sen A. (1987) Sobre ética y economía. Madrid : Alianza Universidad. Segunda reimpresión : 2003.

Sen A. (1992) Inequality Reexamined. New York : Harvard University Press.

\section{Résumé}

\section{Les parcours individuels et la capacité à l'emploi sur les marchés du travail informels : les enseignements de la flexibilité du marché du travail argentin Corina Rodríguez Enríquez}

Le marché du travail argentin se caractérise par le poids du travail informel, marqué par des bas salaires, un fort taux d'instabilité, de mauvaises conditions de travail et peu ou pas de couverture sociale, associés à une instabilité macro-économique. Dans ce contexte, cet article étudie les parcours professionnels des individus et leur impact sur la capacité à l'emploi et le développement des compétences. Une attention particulière est accordée au rôle de l'informalité et le processus d'« informalisation » des emplois formels. Les jeunes, les femmes peu qualifiées et les personnes dont les responsabilités familiales sont importantes, connaissent le plus de difficultés et la plupart de leurs parcours oscillent entre inactivité, chômage et emplois peu qualifiés. Les politiques publiques, comme la mise en place de programmes de formation, ont été incapables de résoudre ces problèmes.

\section{Mots clés}

Marché du travail, flexibilité du marché du travail, sécurisation des parcours professionnels, travail au noir, femme, approche par les capabilités

Journal of Economic Literature: O 17, J 62 